\title{
Uma Abordagem Baseada em Robótica para Ensinar Fundamentos da Computação na Educação Básica
}

\author{
Felipe Oliveira Miranda Cunha ${ }^{1}$, Cristiane Ribeiro do Nascimento ${ }^{2}$ \\ ${ }^{1}$ Universidade Federal da Paraíba (UFPB) - João Pessoa - PB - Brasil \\ ${ }^{2}$ Universidade Estadual da Paraíba (UEPB) - Campina Grande - PB - Brasil \\ \{felipeoliveira.ufpb, cristianeribeiro.ufpb\}@gmail.com
}

\begin{abstract}
This paper presents an approach to teaching the fundamentals of computer science in basic education by robotics. The influence of the approach was evaluated with $3 \mathrm{rd}$, 4th and 5th year elementary students. The results show a better performance among participants who received robotic stimuli.
\end{abstract}

Resumo. Este artigo apresenta uma abordagem para o ensino dos fundamentos da ciência da computação na educação básica por meio da robótica educativa. A influência da abordagem foi avaliada com alunos do $3^{\circ}$, $4^{\circ}$ e $5^{\circ}$ anos do ensino fundamental. Os resultados mostram um melhor desempenho entre os participantes que receberam estímulos da robótica.

\section{Introdução}

Países como Israel [Gal-ezer 1999], Canadá [MEO 2008], Estados Unidos [CSTA 2005] e Inglaterra [Csizmadia et al. 2015] têm defendido o ensino dos fundamentos da computação na educação básica para desenvolver nos estudantes uma maneira computacional de pensar, modelar e resolver problemas de diversas áreas.

No Brasil, a Base Nacional Comum Curricular - BNCC - já inclui em seu texto o desenvolvimento de habilidades alinhadas ao pensamento computacional e à cultura digital [BRASIL 2017a], mas com oportunidades de aprimoramento. Além disso, A BNCC não contempla a inserção da Computação como um componente curricular.

Todavia, a Sociedade Brasileira da Computação - SBC -, por entender que os conceitos da Computação devam ser ensinados a partir do ensino básico, incentiva ações que possam promover a introdução da Computação nas escolas. Em 2017, a SBC elaborou as Diretrizes para ensino de Computação na Educação Básica [SBC 2017]. O documento apresenta as competências gerais e habilidades da Computação no ensino fundamental e médio e defende que a Computação não seja inserida dentro de outras áreas da BNCC, mas sim como objetos de conhecimentos e habilidades que devem ser trabalhados.

O pensamento computacional é essencial para a vida no século XXI e está se tornando um importante aspecto a entrar nos currículos de educação básica. Estudos apontam a possibilidade de inserir o pensamento computacional na educação básica por meio da robótica [Avila et al. 2017] [Oliveira e Araujo 2016], [Da Silva et al. 2016], [Zanetti e Oliveira 2015]. 
VIII Congresso Brasileiro de Informática na Educação (CBIE 2019)

Anais do XXV Workshop de Informática na Escola (WIE 2019)

A robótica é um instrumento capaz de promover o aprendizado dos princípios da Computação [Neto et al. 2015] e de desenvolver o pensamento computacional, fundamental para crianças em idade escolar.

Com base nesse contexto, surgem os seguintes questionamentos: Como definir uma abordagem de ensino da Computação através da robótica visando desenvolver habilidades do pensamento computacional? Quais conceitos básicos da ciência da computação podem ser explorados através dessa abordagem?

Diante disso, este trabalho apresenta uma abordagem baseada em robótica para ensinar os conceitos básicos de Números Binários em Computação na educação básica.

O trabalho está organizado da seguinte forma: a seção 2 descreve os trabalhos relacionados; a seção 3 apresenta a metodologia; a seção 4 descreve os resultados, e; a seção 5 apresenta as conclusões.

\section{Trabalhos relacionados}

Há estudos relevantes na literatura que discorrem acerca de diferentes abordagens para desenvolver o pensamento computacional através da robótica. O estudo de Avila; Cavaleiro [2017] apresenta uma proposta de intervenção com robótica no ensino fundamental baseada em taxonomias de aprendizagem para promover o pensamento computacional por meio da robótica. $O$ estudo sugere que as atividades sejam organizadas nas fases: exposição passiva (sem manipulação), discussão sobre robótica (sem programação), robótica com programação (sem montagem), robótica orientada a engenharia e desafio robótico co-criativo orientado a projetos. $O$ trabalho indica a distribuição do tempo dedicado a cada atividade de acordo com níveis cognitivos da taxonomia (criar, aplicar, lembrar, entender, analisar e avaliar).

O estudo de Ronsivalle et al. [2018] descreve doze habilidades para nortear o professor que ensina através da robótica. O estudo destaca superficialmente os fundamentos da Computação, ao sugerir que esse professor deva ter conhecimento básico em informática, conceitos de usabilidade, de componentes de hardware e de aplicações de tecnologia da informação na sociedade e na escola.

Silva; Javaroni [2018] realizou estudo com alunos do nono ano do ensino fundamental para apoiar a aprendizagem de matemática, especificamente o assunto de Divisão Euclidiana, através de atividades de robótica. Com essa abordagem, o estudo permitiu o desenvolvimento de habilidades do pensamento computacional. O trabalho se desenvolveu inicialmente com a introdução da programação, seguida da familiarização dos alunos com o kit de robótica, para, a partir disso, abordar o conceito específico da matemática. O pensamento computacional foi desenvolvido durante o processo de resolução de problemas matemáticos envolvendo o kit de robótica, tais como a interpretação, abstração e organização lógica de dados.

Já o estudo de Queiroz et al. [2017] investigou o desenvolvimento de habilidades do pensamento computacional com alunos do ensino fundamental I a partir de oficina de programação com robótica, compreendida em 14 aulas. As atividades com robótica envolveram o aprendizado de programação, a construção e programação de modelos robóticos inspirados em animais. Em relação às habilidades do pensamento computacional, foram estimuladas a detecção de erros, a abstração e a construção de 
VIII Congresso Brasileiro de Informática na Educação (CBIE 2019)

Anais do XXV Workshop de Informática na Escola (WIE 2019)

algoritmos. Além disso, o trabalho desenvolveu a compreensão sobre conceitos da ciência da Computação, tais como Estruturas de Controle.

A pesquisa descrita em Souza et al. [2016] explorou habilidades do pensamento computacional por meio da robótica a partir de um curso com estudantes do ensino médio. Após o trabalho desenvolvido, verificou-se que o desempenho dos alunos foi melhorado.

Há estratégias que se preocupam em explorar habilidades do pensamento computacional aliando a robótica com conceitos de dados e de programação. No estudo de Oliveira; Araújo [2016] foram trabalhadas montagens de robôs e desenvolvidas as habilidades de coleta, análise e representação de dados, além da abstração, decomposição, paralelização e simulação. Como resultados, o estudo verificou que a abordagem potencializou as habilidades e competências exploradas nas oficinas e que os alunos conseguiram relacioná-las em atividades cotidianas.

Outras abordagens tendem a motivar os alunos a investigar e a aprender sobre algoritmos. O estudo de Silva et al. [2016] introduziu conceitos de programação e robótica com alunos do fundamental II. Em seguida, aplicou atividades de programação visual e montagem de robô para mostrar a execução de tarefas algorítmicas, como andar, falar, mostrar imagem na tela e ligar leds. Por fim, realizou com os alunos um projeto de semáforo, utilizando plataforma Arduino para montar e programar, de maneira a explorar os conceitos de variáveis e operadores em Computação.

A proposta de Conde et al. [2017] considera a utilização de robôs no ensino dos conceitos de Computação, devido ao potencial motivacional da robótica. O trabalho utilizou um robô de uso industrial para demonstrar aos alunos os conceitos de repetição e condição. Para explicar o conceito de repetição, o robô retira uma bola de uma rampa e a coloca em uma caixa e repete esse movimento mesmo se não houver bolas na rampa. Para explicar o conceito de condição, o robô pega uma bola na mesa e a coloca na caixa, mas, neste caso, somente se houver bola naquele ponto da mesa. Em seguida, os alunos passam a resolver problemas similares. Embora o estudo contribua com as pesquisas na área, a proposta apresenta limitações em relação material utilizado, uma vez que não permite novas configurações na estrutura do robô.

Já o trabalho de Chang et al. [2010] propõe o uso de robôs humanoides para apoio ao ensino de aulas de Matemática como recurso motivador da aprendizagem. Com a abordagem, o estudo recai na problemática de não conseguir estabelecer situações de ensino e aprendizagem da Computação através da robótica.

Diante disso, percebe-se que a comunidade científica já sinaliza uma preocupação no sentido de utilizar a robótica para desenvolver o pensamento computacional. Contudo, grande parte das pesquisas concentram esforços nos anos finais do fundamental e no nível médio. Entretanto, é preciso garantir que os princípios básicos da ciência da Computação sejam trabalhados nos anos iniciais do ensino fundamental. Ter um olhar computacional para resolver problemas nos primeiros anos de formação escolar pode contribuir para que as gerações futuras sejam capazes de construir soluções inovadoras e impulsionar o cenário de pesquisa científica no Brasil e, com isso, torná-lo protagonista no cenário mundial. 
VIII Congresso Brasileiro de Informática na Educação (CBIE 2019)

Anais do XXV Workshop de Informática na Escola (WIE 2019)

\section{Abordagem proposta}

A inserção da robótica foi pensada como um recurso instrucional que promova o efetivo entendimento dos conceitos expostos, tendo em vista a dificuldade que os alunos têm para a assimilação e conversão de números binários, que foi verificada após aulas expositivas.

A abordagem foi aplicada com diferentes turmas do $3^{\circ}, 4^{\circ}$ e $5^{\circ}$ anos do ensino fundamental em diferentes escolas para verificar até que ponto o uso da robótica influencia na desenvoltura dos estudantes ao encarar problemas de conversão binária de níveis de dificuldade distintos. Na aplicação, cada turma foi dividida em dois grupos de forma aleatória. O primeiro grupo participou da atividade desplugada e, em seguida, resolveu problemas de conversão binária. $\mathrm{O}$ segundo grupo participou da atividade desplugada e da experimentação do robô para, em seguida, resolver questões de conversão binária.

\subsection{Explicação sobre o assunto}

A explicação do tópico Números Binários aconteceu durante uma aula para todas as turmas. Para melhor compreensão dos alunos sobre o assunto, utilizou-se cartões contendo imagens de lâmpadas acesas e apagadas, associando-as aos conceitos de bit ligado e bit desligado, respectivamente, e que a partir de combinações desses estados é possível representar números e outros dados. Durante a explicação, foram dados alguns exemplos de números aleatórios para que os alunos realizassem a conversão bináriodecimal e decimal-binária, conforme Figura 1.

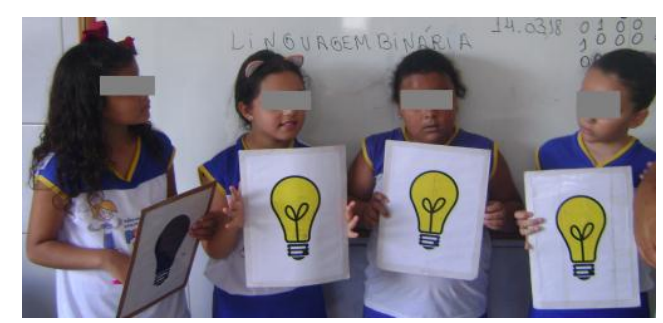

Figura 1. Participação dos alunos na explicação.

Ao final, os alunos foram divididos em dois grupos de tamanho igual.

\subsection{Conversão Binária com o Robô}

Nesse momento, apenas um grupo utilizou o robô para realizar conversão bináriodecimal. Os alunos experimentaram o robô com testes de números binários aleatórios, como forma de receber o estímulo da robótica na compreensão do assunto.

A conversão com o robô funciona da seguinte forma: $\mathrm{O}$ robô programado na linguagem NXC, versão baseada em $\mathrm{C}$, utiliza sensores de toque para acionamento dos bits e motores, bem como lâmpadas, para ativação dos zeros e uns para formar o Byte. Quando um sensor é ativado ou desativado, o motor correspondente gira $180^{\circ}$ ou $-180^{\circ}$, respectivamente, e apresenta o número binário correspondente em pequenos cartões. Assim, o número decimal equivalente às combinações de números binários é exibido na tela do robô, conforme Figura 2. 
VIII Congresso Brasileiro de Informática na Educação (CBIE 2019)

Anais do XXV Workshop de Informática na Escola (WIE 2019)

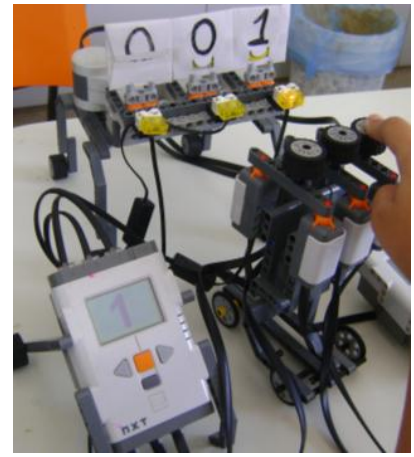

Figura 2. Robô que executa a conversão binária.

\subsection{Resolução de problemas de conversão binária}

A atividade contemplou problemas de conversão a partir da contagem de pontos em cartas para valores decimais ( $\mathrm{CP} 1$ e $\mathrm{CP} 2)$, de conversão de símbolos de lâmpadas ligada e desligada para valores em decimal (L1 e L2), da conversão binário-decimal (BD1 e $\mathrm{BD} 2$ ) e outros quatro problemas que envolveu a conversão decimal-binário (DB1, DB2, DB3 e DB4). Um exemplo da atividade é ilustrada na Figura 3.

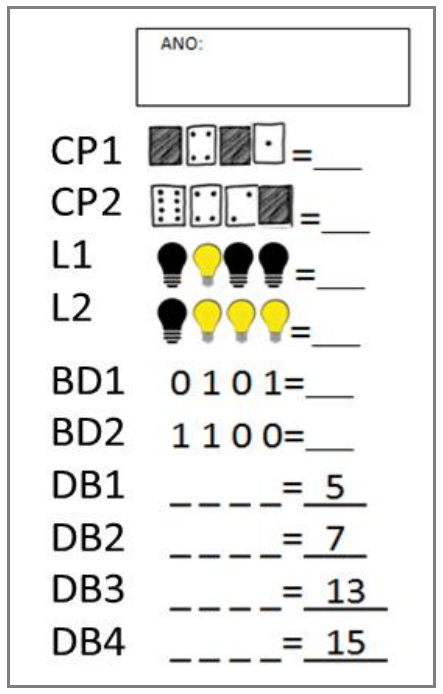

Figura 3. Atividade de resolução de conversão binária.

Essa atividade foi aplicada com 59 estudantes do $3^{\circ}$ ano, 49 estudantes do $4^{\circ}$ ano e 30 estudantes do $5^{\circ}$ ano do ensino fundamental $1 \mathrm{em}$ diferentes escolas privadas.

\section{Resultados}

Dos 59 estudantes do $3^{\circ}$ ano que participaram do estudo, 29 não receberam o estímulo da robótica, enquanto que 30 alunos receberam o estímulo. O grupo de estudantes do $3^{\circ}$ ano que não receberam estímulo do robô obteve bom desempenho nas atividades que demandavam a contagem dos pontos para responder o valor decimal, CP1 e CP2, bem como a atividade de verificar a posição das lâmpadas acesas e apagadas, L1. Entretanto, após a questão L2, o índice de erros aumentou, chegando a 25 na resolução da conversão binário-decimal, B-D2, e o número de acertos caiu para 5 na mesma questão, conforme Figura 4. 
VIII Congresso Brasileiro de Informática na Educação (CBIE 2019)

Anais do XXV Workshop de Informática na Escola (WIE 2019)

Já o grupo de estudantes do $3^{\circ}$ ano que recebeu o estímulo da robótica, apresentou melhores resultados no teste de conversão em relação ao grupo que não interagiu com o robô. Isso foi percebido quando os índices de acertos se mantiveram entre 23 e 30, bem como os índices de erros se mantiveram entre 1 e 7, Figura 4.
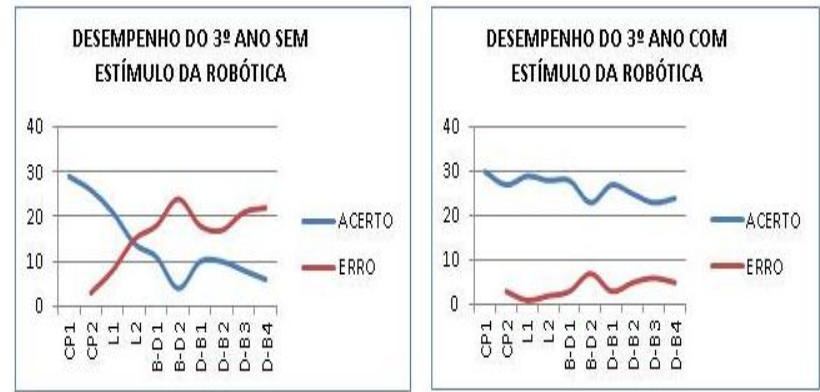

Figura 4. Desempenho dos alunos do 3 ano.

Em relação ao $4^{\circ}$ ano, dos 49 estudantes que participaram do estudo, 23 não receberam o estímulo da robótica, enquanto que 26 alunos receberam o estímulo. Analisando o desempenho do grupo de estudantes do $4^{\circ}$ ano que não receberam estímulo do robô, foi possível perceber uma grande dificuldade, refletida no baixo número de acertos, com relação às atividades L2, B-D2 e D-B2, conforme Figura 4.

Já o desempenho do grupo de estudantes do $4^{\circ}$ ano que recebeu estímulos da robótica foi melhor em relação ao grupo que não recebeu. Isso é evidente, pois os índices de acertos e de erros se mantiveram entre 20 a 26 e 1 a 4, respectivamente, conforme Figura 5.

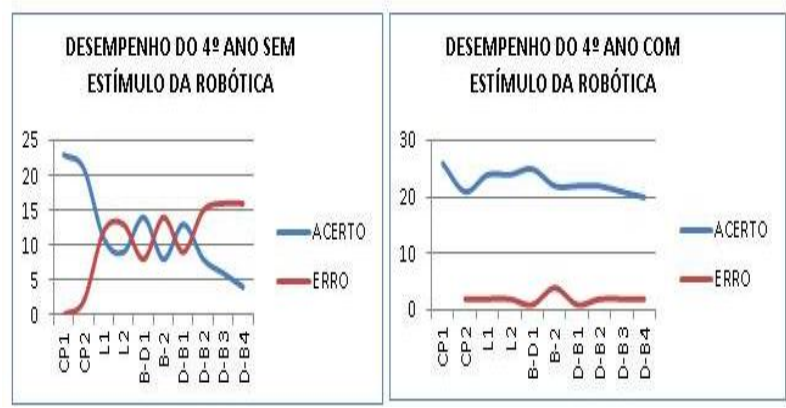

Figura 5. Desempenho dos alunos do $4^{\circ}$ ano.

Já sobre o grupo de estudantes do $5^{\circ}$ ano, do total de 30 estudantes participantes, 16 receberam o estímulo da robótica, enquanto que 14 não receberam o mesmo estímulo. Em relação ao grupo que não recebeu estímulos da robótica, 14 estudantes, o desempenho foi bom apenas nas questões CP1 e CP2, pois, a partir dessas, o nível de acerto diminuiu a 5 acertos nas questões que envolviam a conversão decimal-binária.

Por outro lado, o desempenho do grupo do $5^{\circ}$ ano que recebeu estímulos da robótica foi melhor. $\mathrm{O}$ gráfico ilustrado na Figura 6 mostra que o número de acertos se manteve entre 11 e 16, bem acima em relação ao grupo que não obteve o estímulo da robótica. 
VIII Congresso Brasileiro de Informática na Educação (CBIE 2019)

Anais do XXV Workshop de Informática na Escola (WIE 2019)

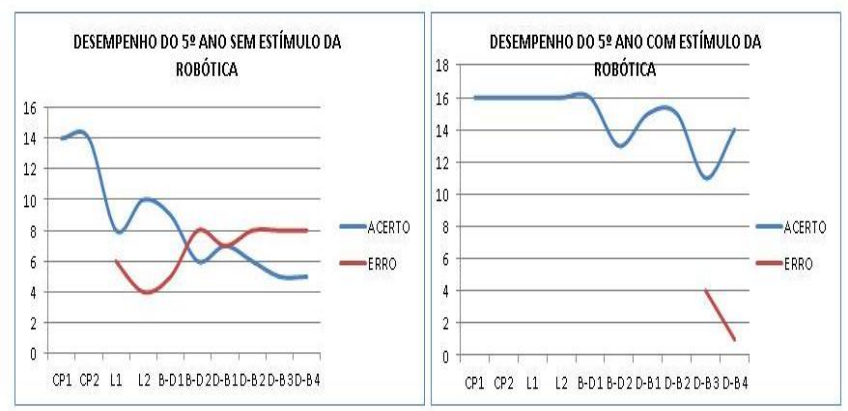

Figura 6. Desempenho dos alunos do $5^{\circ}$ ano.

\section{Conclusões}

Este trabalho apresenta uma abordagem de importante contribuição à comunidade científica na área de estudo, tendo em vista discutir possibilidades de introduzir o pensamento computacional na escola e de desenvolver habilidades e competências fundamentais para este século.

Buscou-se, analisar, refletir e compreender as potencialidades do uso da robótica na sala de aula como recurso didático no processo de aprendizagem em Computação e no estímulo às habilidades do pensamento computacional.

Em relação aos resultados do estudo, foi possível indicar pontos a serem reforçados no aprendizado sobre tópicos da ciência da computação, aliando o ensino por atividades desplugadas e a robótica educativa, e promover o aprendizado efetivo de conceitos complexos de serem compreendidos apenas com aulas expositivas.

A aplicação da abordagem permitiu perceber e reforçar a necessidade de se trabalhar conteúdos correlatos da matemática ao mesmo tempo em que se aprende os conceitos computacionais, além de possibilitar o incentivo ao aprendizado tecnológico e o desenvolvimento de importantes habilidades, tais como a associação, a abstração e o uso de símbolos e metáforas.

\section{Referências}

Andrade, D., Carvalho, T., Silveira, J., Cavalheiro, S., Foss, L., Fleischmann, A. M., Aguiar, M., Reiser R. Proposta de Atividades para o Desenvolvimento do Pensamento Computacional no Ensino Fundamental, CBIE Trilha WIE 2013.

Avila, C., Cavalheiro, S., Bordini, A., Marques, M. O Pensamento Computacional por meio da Robótica no Ensino Básico - Uma Revisão Sistemática. In: XXVIII Simpósio Brasileiro de Informática na Educação SBIE (Brazilian Symposium on Computers in Education), 2017, Recife, 2017. p. 82-91.

Avila, C.. Cavalheiro, S. (2017). Robótica Educacional como Estratégia de Promoção do Pensamento Computacional - Uma Proposta de Metodologia Baseada em Taxonomias de Aprendizagem. Anais dos Workshops do VI Congresso Brasileiro de Informática na Educação (WCBIE 2017).

Bebras. Disponível em : <https://www.Bebras.org/?q=about $>$. Acesso em: 05 de Janeiro de 2019. 
VIII Congresso Brasileiro de Informática na Educação (CBIE 2019)

Anais do XXV Workshop de Informática na Escola (WIE 2019)

Bell, T.; Whitten, I.; Fellows, M. Computer Science Unplugged.Universidade de Canterbury, Nova Zelândia, 2007.105 p.

BRASIL. Base Nacional Comum Curricular. 2017a. Disponível em: $<$ http://agbcampinas.com.br/site/http://agbcampinas.com.br/site/wpcontent/uploads/2 017/08/BNCC_publicacao.pdf $>$. Acesso em: 25 jan. 2019.

Brennan, K.; Resnick, M.New frameworks for studying and assessing the development of computational thinking.AERA 2012, Vancouver, Canadá, 2012.

Chang, C.W.; Lee, J.-H.; Chao, P.Y.; Wang, C.Y.; Chen, G.-D. (2010). Exploring the Possibility of Using Humanoid Robots as Instructional Tools for Teaching a Second Language in Primary School. Educational Technology \& Society,13(2),13-24.

Cohen, L., Manion, L., and Morrison, K. (2011).Research Methods in Education.Education, Research methods.Routledge.

Conde, M. Á.; Fernández-Llamas, C.; Rodríguez-Sedano, Francisco J.; GuerreroHigueras, Á. M.; Matellán-Olivera, V.; García-Peñalvo, F. J. (2017) Promoting Computational Thinking in K-12 students by applying unplugged methods and robotics, Proceedings of the 5th International Conference on Technological Ecosystems for Enhancing Multiculturality, p.1-6, October 18-20,2017, Cádiz, Spain.

Computer Science Teacher Association CSTA. (2005) The New Educational Imperative: Improving High School Computer Science Education. Final Report of the CSTA.Curriculum Improvement Task Force. ACM - Association for Computing Machinery.

Csizmadia, A.; Curzon, P.; Dorling, M.; Humphreys, S.; Thomas Ng; Selby, C.; Woollard, J.. (2015). Computationalthinking: a guide for teachers. Computing At School.

Cunha, F. O. M.; Nascimento, C. R. (2018) Uma Abordagem Baseada em Robótica e Computação Desplugada para Desenvolver o Pensamento Computacional na Educação Básica. In Anais do Simpósio Brasileiro de Informática na Educação 2018.

Dagiene, Valentina, Sentance, Sue; Stupuriene Gabriele, Bebras-a sustainable community building model for the concept based learning of informatics and computational thinking. Informatics in Education-An International Journal, (Vol15_1): 25-44,2016.

De Paula, B. H.; Valente, J. A.; Burn, A. O uso de jogos digitais para o desenvolvimento do currículo para a Educação Computacional na Inglaterra. Currículo semFronteiras, v. 14 , n. 3, p. 46-71, set/dez 2014.

Gal-Ezer, J.; D. Harel.(1999) Curriculum for a high school computer science curriculum.Computer Science Education 9(2).

Guedes, Aníbal Lopes; Kerber, Fábio Matias. Usando a robótica como meio educativo. Unoesc \& Ciências “ACET, Joaçaba, v.1, n.2, p.199-208, jul./dez. 2010.

Ministério da Educação de Ontário - MEO. (2008). "Currículo para o ensino de Ciência da Computação nas escolas". Retirado de www.edu.gov.on.ca. 
VIII Congresso Brasileiro de Informática na Educação (CBIE 2019)

Anais do XXV Workshop de Informática na Escola (WIE 2019)

Neto, R. P. B., Santana, A. M., Rocha, D. P.; Souza, A. (2015). Robótica na educação: Uma revisão sistemática dos últimos 10 anos. In Anais do SBIE 2015, p. 386-395.

Oliveira, E.;Araujo, A. L. (2016). Pensamento Computacional e robótica: Um estudo sobre habilidades desenvolvidas em oficinas de robótica educacional. In Anais do SBIE 2016, páginas 530-539.

Petersen, K.; Feldt, R.; Mujtaba, S.; Mattsson, M.; Systematic mapping studies in software engineering. In: XII International Conference On Evaluation And Assessment In Software Engineering, p. 68-77, 2008.

Queiroz, R. L; Sampaio, F. F; Santos, M. P. Pensamento Computacional, robótica e educação. Tecnologias, Sociedade e Conhecimento, Campinas, v. 4, n. 1, Dez. 2017.

Ronsivalle, Gaetano Bruno \&Boldi, Arianna\&Gusella, V \&Inama, C \& Carta, Simona. (2018). How to Implement Educational Robotics' Programs in Italian Schools: A Brief Guideline According to an Instructional Design Point of View. Technology, Knowledge and Learning.

SBC. Diretrizes para ensino de Computação na Educação Básica. 2017. Disponível em: http://www.sbc.org.br/documentos-da-sbc/send/131-curriculos-de-referencia/1177diretrizes-para-ensino-de-computacao-naeducacao-basica. Acesso em: mar. de 2019.

Silva, D. P. et al. (2016). Aplicação de robótica na educação de forma gradual para o estímulo do pensamento computacional. In Anais dos Workshops do CBIE 2016, páginas 1188-1197.

Silva E.;Javaroni S. L. (2018) Pensamento Computacional e atividades com robótica para a promoção da aprendizagem sobre o significado do resto da divisão euclidiana. In Anais do SimpósioBrasileiro de Informática na Educação - SBIE 2018.

Wing, J. M. (2006).Computational thinking.Commun. ACM, 49(3):33-35.

Wohlin, C., Runeson, P., Host, M., Ohlsson, M. C., Regnell, B., Wesslen A., Experimentation in software engineering. Springer Science \& Business Media; 2012.

Zanetti, H.; Oliveira, C. (2015). Praticas de ensino de programação de computadores com robótica pedagógica e aplicação de pensamento computacional. In Anais dos Workshops do CBIE, páginas 1236-1245. 